D. Popescu

Nagoya Math. J.

Vol. 113 (1989), 121-128

\title{
POLYNOMIAL RINGS AND THEIR PROJECTIVE MODULES
}

\author{
DORIN POPESCU
}

\section{$\S 1$. Introduction}

Let $R$ be a regular noetherian ring. A central question concerning projective modules over polynomial $R$-algebras is the following.

(1.1) Bass-Quillen Conjecture ([2] Problem IX, [10]). Every finitely generated projective module $F$ over a polynomial $R$-algebra $R[T], T=\left(T_{1}\right.$, $\left.\cdots, T_{n}\right)$ is extended from $R$, i.e.

$$
P \cong R[T] \otimes_{R} P /(T) P .
$$

When $\operatorname{dim} R \leqslant 1$ the conjecture is true by the Quillen-Suslin Theorem (see [10], [13]). Also H. Lindel [5] proved that the conjecture is true for regular rings essentially of finite type over a field. His ideas work also in some mixed characteristic cases (cf. [7]; see also Proposition (2.1) and Theorem (4.1) below which we included here for the sake of completeness). These results show that it is worth to consider the following

(1.2) Question ([7]). A regular local ring is a filtered inductive limit of regular local rings essentially of finite type over $Z$.

In [8] Corollary (2.7) we stated (1.2) in the equal characteristic case which gave us the possibility to solve in [9] (using [3]) a question of Quillen (see [10]) (and so the BQ Conjecture) in the equal characteristic case.

In this paper we obtain some results in the mixed characteristic case concerning these two questions. Our Theorem (3.1) says that (1.2) is true for a regular local ring $(A, \mathfrak{m})$ when either $p:=\operatorname{char}(A / m) \notin \mathfrak{m}^{2}$, or $A$ is excellent henselian. Using Lindel's results we solve the BQ Conjecture for regular noetherian rings $R$ such that for every prime integer $t$, either $t$ is an unit in $R$, or $R / t R$ is a regular ring (see Corollary (4.3)). If $p \in \mathrm{m}^{2}$ our Corollary (2.4) and Theorem (3.1) iii) show that it is "almost" enough

Received July 9, 1987. 
to prove the $\mathrm{BQ}$ Conjecture for rings of the type $B[X]_{(q, X)} /(f, h)$, where $(B, q)$ is a localization of a polynomial $Z$-algebra, $f, h \in B[X], h$ is monic (in $X$ ) with $h(0) \in q$ but $(\partial h / \partial X)(0) \notin q$ and $f \in p+(q, X)^{2} B[X]_{(q, X)}$.

We would like to thank Professor M. P. Murthy who kindly sent us Swan's notes on Lindel's results and to Professor R. A. Rao who also gave us useful informations on this subject.

\section{$\S 2$. The structure of regular local rings essentially of finite type over $Z$}

(2.1) Proposition (Lindel, [5] and [7]). Let $A$ be a DVR (i.e. noetherian discrete valuation ring), $p$ a local parameter in $A,(R, \mathfrak{m})$ a regular local A-algebra essentially of finite typ $, K:=R / \mathfrak{m}$ and $k=F r(A / \mathfrak{m} \cap A)$. Suppose that

i) $k \subset K$ is separable,

ii) $p \notin \mathfrak{m}^{2}$

iii) $\operatorname{dim} R \geqslant 2$.

Then $R$ is an etale neighbourhood of a localization of a polynomial $A$ algebra.

Proof. Let $u=\left(u_{1}, \cdots, u_{s}\right)$ be a system of elements from $R$ inducing a separable transcendence base of $K$ over $k$. Then changing $A$ by $A[u]_{p A[u]}$ we can reduce our question to the case when $k \subset K$ is finite separable. Thus $R$ is a localization of a finite type $A$-algebra $C$ in a maximal ideal $q \subset C$. Suppose $p \in \mathfrak{m}$, otherwise suppress $p$ from below.

Let $w \in C$ be an element inducing a primitive one $\bar{w}$ for $k \subset K$. If $A \subset R$ is algebraic then $R$ is DVR which is not the case. Thus we can choose $w$ to be transcendental over $A$. Let $f$ be a monic polynomial from $A[W]$ lifting $\operatorname{Irr}(k, \bar{w})$. By separability we have $(\partial f / \partial W)(w) \notin \mathfrak{m}$. If $f(w)$ $\epsilon\left(p, \mathfrak{m}^{2}\right)$ then choose an element $w^{\prime}$ in $q$ which is not in $\left(p, \mathfrak{m}^{2}\right)(\operatorname{dim} R \geqslant 2)$. Then

$$
f\left(w+w^{\prime}\right) \equiv w^{\prime}(\partial f / \partial W)(w) \not \equiv 0 \bmod \left(p, \mathfrak{m}^{2}\right)
$$

and changing $w$ by $w+w^{\prime}$ can suppose that $f(w) \notin\left(p, \mathfrak{m}^{2}\right)$. Choose some elements $x_{2}, \cdots, x_{n}$ in $q, n=\operatorname{dim} R-1$ inducing a base in $\mathfrak{m} /\left(p, f(w), \mathfrak{m}^{2}\right)$ and consider the $\operatorname{map} \varphi: A[X] \rightarrow C, X=\left(X_{1}, \cdots, X_{n}\right) \sim\left(w, x_{2}, \cdots, x_{n}\right)$. By [6] Theorem $81, \varphi$ gives faithfully flat map

$$
R^{\prime}:=A[X]_{\left(p, f\left(X_{1}\right), x_{2}, \ldots, x_{n}\right)} \stackrel{\psi}{\longrightarrow} R
$$


It remains to show that $\psi$ is etale $\left(R, R^{\prime}\right.$ have the same residue field). Applying Zariski Main Theorem ([11], chap. IV, Theorem 1) to $\varphi, q$ we get that $R$ is a localization of a finite $A[X]$-algebra. Since $\psi$ is faithfully flat we are through.

(2.2) Lemma. Let $\left(R^{\prime}, \mathfrak{m}^{\prime}\right) \stackrel{f}{\longrightarrow}(R, \mathfrak{m})$ be a surjective morphism of regular local algebras over a DVR $A$ and $p$ a local parameter in $A$. Suppose that $p \notin \mathfrak{m}^{\prime 2}$ but $p \in \mathfrak{m}^{2}$. Then there exist a regular local ring $\left(R^{\prime \prime}, \mathfrak{m}^{\prime \prime}\right)$ and two surjective morphisms $g: R^{\prime} \rightarrow R^{\prime \prime}, h: R^{\prime \prime} \rightarrow R$ such that

i) $p \notin \mathfrak{m}^{\prime \prime 2}$

ii) $\operatorname{dim} R^{\prime \prime}=\operatorname{dim} R+1$,

iii) $f=h g$,

iv) $\operatorname{Ker} h$ is generated by one element from $p+\mathfrak{m}^{\prime 2}$.

Proof. Let $q=\operatorname{Ker} f$. By hypothesis $p \in q+\mathfrak{m}^{\prime 2}$ and so there exists $x_{1} \in q$ such that $x_{1} \equiv p \bmod \mathrm{m}^{\prime 2}$. Complete $x_{1}$ with some elements $x_{2}, \cdots, x_{s}$ $\epsilon q, s:=\operatorname{dim} R^{\prime}-\operatorname{dim} R$ in order to induce a base $\bar{x}$ in $q+\mathfrak{m}^{\prime 2} / \mathfrak{m}^{\prime 2}$. Then $x$ generates $q$ by [6] Theorem 36. Clearly $R^{\prime \prime}:=R^{\prime} /\left(x_{2}, \cdots, x_{s}\right)$ is the wanted regular local ring and $f$ factorises through it.

(2.3) Proposition. Let $A$ be a $D V R, p$ a local parameter in $A,(R, \mathfrak{m})$ a regular local A-algebra essentially of finite type, $K:=R / \mathfrak{m}$ and $k:=$ $\operatorname{Fr}(A / \mathrm{m} \cap A)$. Suppose that

i) $k \subset K$ is separable,

ii) $p \in \mathfrak{m}^{2}$,

iii) $\operatorname{dim} R \geqslant 2$.

Then there exists a localization $(B, \mathfrak{n})$ of a polynomial A-algebra and two polynomials $f, h \in B[T]$ such that

1) $R \cong B[T]_{(n, T)} /(f, h)$ as A-algebras

2) $h(0) \in \mathfrak{n},(\partial h / \partial T)(0) \notin \mathfrak{n}, h$ monic

3) $f \in p+(\mathfrak{n}, T)^{2} B[T]_{(\mathfrak{n}, T)}$.

Proof. Since $R$ is essentially of finite type over $A$ we can express $R$ as factor algebra of a localization $\left(R^{\prime}, \mathfrak{m}^{\prime}\right)$ of a polynomial $A$-algebra. Using Lemma (2.2) we express $R$ as a factor of a regular local $A$-algebra $\left(R^{\prime \prime}, \mathfrak{m}^{\prime \prime}\right)$ essentially of finite type with $p \notin \mathfrak{m}^{\prime 2}$ through an ideal generated by an element $f$ from $p+\mathfrak{m}^{\prime \prime 2}$. By Proposition (2.1). $R^{\prime \prime}$ is an etale neighbourhood of a localization $(B, \mathfrak{n})$ of a polynomial $A$-algebra. Thus 
$R^{\prime \prime} \cong B[T]_{(\mathfrak{n}, T)} /(h)$ for a polynomial $h$ satisfying (2) (see [11] chap. V, Theorem 1).

(2.4) Corollary. Let $(R, \mathfrak{m})$ be a regular local ring essentially of finite type over $Z$ and $p:=\operatorname{char}(R / \mathfrak{m})$. Then there exist a localization $(B, \mathfrak{n})$ of a polynomial Z-algebra and two polynomials $f, h \in B[T]$ such that

i) $R \cong B[T]_{(\mathfrak{n}, T)} /(f, h)$,

ii) $h(0) \in \mathfrak{H},(\partial h / \partial T)(0) \notin \mathfrak{n}, h$ monic,

iii) $f \in p+(\mathfrak{n}, T)^{2} B[T]_{(\mathfrak{n}, T)}$ if $p \in \mathfrak{m}^{2}$ and $p \cdot 1_{R} \neq 0$; otherwise $f=0$.

For the proof apply Propositions (2.1) and (2.3) for $A=Z_{(p)}$.

(2.5) Remark. Looking to the above Corollary one can ask if all regular local rings essentially of finite type over $Z$ are etale neighbourhoods of rings of type $B /(f)$. The answer seems to be negative.

Let $R:=Z[X, T]_{(p, X, T)} /(f, h)$, where $f:=p-X^{2}(1+T)$ and $h:=T^{2}$ $+2 T-X^{2}$ for $p \neq 2$. Since $(f) \cap Z[X]_{(p, X)}=\left(p^{2}-X^{4}-X^{6}\right) \in(p, X)^{2}$, the ring $Z[X]_{(p, X)} /\left(p^{2}-X^{4}-X^{6}\right)$ is not regular and so $R$ is not an etale neighbourhood of a factor ring of $Z[X]_{(p, X)}$.

\section{§ 3. Reduction to regular local rings essentially of finite type over $\mathrm{Z}$}

(3.1) Theorem. A regular local ring $(R, \mathfrak{m})$ is a filtered inductive limit of regular local rings essentially of finite type over $Z$ if either

i) $p:=\operatorname{char}(R / \mathfrak{m}) \notin \mathfrak{m}^{2}$, or

ii) $R$ contains a field, i.e. $p \cdot 1_{R}=0$, or

iii) $R$ is excellent henselian.

Proof. When $R$ contains a field we need just [8] Corollary (2.7). If $p \notin \mathfrak{m}^{2}$ then the inclusion $Z_{(p)} \longleftrightarrow R$ is formally smooth by [4] (19.7.1) and so a regular map because $Z_{(p)}$ is excellent (see [6] (34.B)). Applying [8] Theorem (2.5) we can express $R$ as a filtered inductive limit of smooth local $Z_{(p)}$-algebras essentially of finite type which are certainly regular local rings ([6] (33.B)).

For iii) we first assume that $R$ is a complete local ring with $p \in \mathfrak{m}^{2}$, $p \cdot 1_{R} \neq 0$. By the Cohen Structure Theorem $R$ is a factor of a complete regular local ring $(A, \mathfrak{n})$ with $p \notin \mathfrak{n}^{2}$. Using (i) we express $A$ as a filtered inductive limit of regular local rings essentially of finite type over $Z$ and so it is enough to apply the following:

(3.2) LEMMA. Let $(A, \mathfrak{n}) \rightarrow(R, \mathfrak{m})$ be a surjective morphism of regular 
local rings. Suppose that $(A, \mathfrak{n})$ is a filtered inductive limit of regular local rings essentially of finite type over $Z$. Then $(R, \mathfrak{m})$ is also a filtered inductive limit of regular local rings essentially of finite type over $Z$.

Suppose now that $R$ is an excellent (non complete) henselian local ring. Then $R$ has the property of Artin approximation by [8] Theorem (1.3) i.e. in particular every finite system of polynomial equations over $R$ has a solution in $R$ iff it has one in its completion $\hat{R}$. It is enough to prove that for every finite type sub- $Z$-algebra $B$ of $R$ the inclusion $B \hookrightarrow R$ can be factorised through a regular local $Z$-algebra $C$ essentially of finite type as shows the following Lemma-a variant of [1] Lemma (5.2):

(3.3) LEMMA. The following statements are equivalent:

i) $R$ is a filtered inductive limit of regular local rings essentially of finite type over $Z$,

ii) for every finite type sub-Z-algebra $B$ of $R$ the inclusion $B \hookrightarrow R$ can be factorised by a regular local ring essentially of finite type over $Z$.

Fix a finite type sub-Z-algebra $B$ of $R$. As above $\hat{R}$ is a filtered inductive limit of regular local $Z$-algebras essentially of finite type. Then there exists a regular local $Z$-algebra $\tilde{C}$ essentially of finite type and two morphisms $\tilde{u}: B \rightarrow \tilde{C}, \hat{v}: \tilde{C} \rightarrow \hat{R}$ such that the following diagram commutes

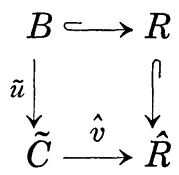

Choose a finite type sub- $Z$-algebra $D$ of $\tilde{C}$ and a prime ideal $q \subset D$ such that

1) $\tilde{C} \cong D_{q}$

2) $\tilde{u}$ factorises through $D$, let us say $\tilde{u}$ is the composite $\operatorname{map} B \stackrel{u}{\longrightarrow}$ $D \rightarrow D_{q} \cong \tilde{C}$.

By [6] (34.A) $D$ is an excellent ring and so Reg $D$ is open. In particular $D_{d}$ is a regular ring for a certain $d \notin q$. Changing $D$ by $D^{\prime}$ we can suppose $D$ regular. The composite map $\tilde{v}: D \rightarrow D_{q} \cong \tilde{C} \rightarrow \hat{R}$ makes commutative the following diagram

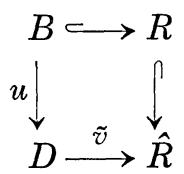


The existence of $\tilde{v}$ such that the above diagram is commutative means in other words that a certain system of polynomial equations over $R$ has a solution in $\hat{R}$. Indeed let $D \cong Z[X] /(h), X=\left(X_{1}, \cdots, X_{n}\right), h=\left(h_{1}, \cdots, h_{s}\right)$, the isomorphism being given by $X \backsim x \in D^{n}$. Choose a system of generators $b=\left(b_{1}, \cdots, b_{t}\right)$ of $B$ as $Z$-algebra and let $P=\left(P_{1}, \cdots, P_{t}\right)$ be some polynomials from $Z[X]$ lifting $u(b)$ modulo $h$. Clearly the wanted system is the following

$$
\left\{\begin{array}{l}
P(X)=b \\
h(X)=0
\end{array}\right.
$$

Since (*) has a solution in $\hat{R}$ (given by $\hat{v}$ ) it has also one in $R$ because $R$ has the property of Artin approximation. Thus there exists $v: D \rightarrow R$ such that the following diagram commutes

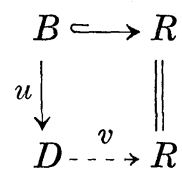

Then $C:=D_{v-1_{\mathfrak{m}}}$ works.

Proof of Lemma (3.2). Let $a$ be the kernel of $A \rightarrow R$. Then $\mathfrak{a}$ is generated by a system of $s:=\operatorname{dim} A-\operatorname{dim} R$ elements $x$ which is a part of a regular system of parameters from $A$ (see [6] Theorem 36). By hypothesis $A$ is a filtered inductive limit of regular local $Z$-algebras $\left(A_{i}, \mathfrak{n}_{i}\right)$, $i \in I$ essentially of finite type. Clearly we can suppose that for every $i \in I$ $x$ is the image of a system of elements $x_{i} \in A_{i}^{s}$ by $A_{i} \rightarrow A$. Since $x$ gives a linearly independent system in $\mathfrak{n} / \mathfrak{n}^{2}$ it follows that $x_{i}$ induces a linearly independent system in $\pi_{i} / \mathfrak{n}_{i}^{2}$ for $i$ high enough. Thus we can restrict our limit to the case when $x_{i}$ is a part of a regular system of parameters of $A_{i}$ for every $i \in I$. Then $R_{i}:=A_{i} / x_{i} A_{i}, i \in I$ are regular local rings and we have $R \cong \underline{\lim }_{i \in I} R_{i}$.

(3.4) Lemma. Let $A$ be a Dedekind ring and $R$ a regular (noetherian) A-algebra such that for every prime ideal $q \subset A$ with $q R \neq R$

i) $\operatorname{Fr}(A / q)$ is a perfect field,

ii) $R / q R$ is regular.

Then $R$ is a filtered inductive limit of finite type regular A-algebras.

Proof. First note that the structural map $A \rightarrow R$ is regular. Let $q \subset R$ be $a$ prime ideal. Then $\varphi_{q}: A_{q \cap A} \rightarrow R_{q}$ is a faithfully flat map 
because $A_{q \cap A}$ is a field or a DVR and $R_{q}$ is a domain. By i) and ii) $\varphi_{q}$ is formally smooth (see [4] (19.7.1)). Using [8] Theorem (2.5) we can express $R$ as a filtered inductive limit of finite type smooth $A$-algebras which are certainly regular (see [6] (33.B)).

(3.5) Remark. The problem to apply [8] Theorem (2.5) in order to solve Conjecture (1.2) (see the proof of Theorem (3.1)) raises the question to study the regular local rings $(R, \mathfrak{m})$ containing a regular local $Z$-algebra $(A, \mathfrak{n})$ essentially of finite type such that

i) the inclusion $A \subset R$ is flat,

ii) $\mathfrak{m}=\mathfrak{n} R$.

\section{§4. On the Bass-Quillen Conjecture}

(4.1) Theorem (Lindel [5] and [7]). Let $A$ be a Dedekind ring and $R$ a regular A-algebra of finite type. Suppose that for every prime ideal $q \subset A$ with $q R \neq R$

i) $\operatorname{Fr}(A / q)$ is a perfect field,

ii) $R / q R$ is regular.

Then the BQ Conjecture holds for $R$.

Proof. Apply induction on $n:=\operatorname{dim} R$. By Quillen's Patching Theorem ([10] Theorem 1) we can assume $A$ and $(R, \mathfrak{m})$ local. Then $A$ is a field or a DVR. If $A$ is a field then choose an element $x \in \mathfrak{m} \backslash \mathfrak{m}^{2}$ (we may suppose $n \geqslant 2$ by Quillen-Suslin Theorem). Changing $A$ by $A[x]_{(x)}$ we reduce to the case when $A$ is a DVR. By Proposition (2.1), $R$ is an etale neighbourhood of a localization $B$ of a polynomial algebra $C$ over $A$.

Le $P$ be a finitely generated projective module over a polynomial $R$ algebra $R[T], T=\left(T_{1}, \cdots, T_{s}\right)$. By the induction hypothesis we can suppose that $P_{h} \simeq R_{h}[T] \otimes_{R_{h}}\left(P_{h} /(T) P_{h}\right)$ for all $h \in \mathfrak{m} \cap B$. Since $P /(T) P$ is free ( $R$ is local) we get $P_{h}$ free. Then $P$ is an extension of a finitely generated projective $B[T]$-module $P^{\prime}$ by the Corollary from [5]. But all finitely generated projective $C[T]$-modules are extended from $C$ (in fact from $A$ by Quillen-Suslin Theorem). Then all finitely generated projective $B\left[T^{\prime}\right]$-modules are extended from $B$ by [12] Proposition 2. Thus $P^{\prime}$ is free ( $B$ is local) and so $P$ is free too.

(4.2) Theorem. Let $A$ be a Dedekind ring and $R$ a regular (noetherian) $A$-algebra such that for every prime ideal $q \subset A$ with $q R \neq R$ 
i) $\operatorname{Fr}(A / q)$ is a perfect field,

ii) $R / q R$ is regular.

Then the BQ Conjecture holds for $R$.

Proof. By Lemma (3.4) $R$ is a filtered inductive limit of finite type regular $A$-algebras, let us say $R \cong \varliminf_{i \in I} R_{i}$. Let $P$ be a finitely generated projective $R[T]$-module, $T=\left(T_{1}, \cdots, T_{s}\right)$. Then $P$ is extended from $R_{i}[T]$ for a certain $i \in I$ because $R[T]=\varliminf_{i m} R_{i}[T]$. But BQ Conjecture holds for $R_{i}$ by Theorem (4.1). Thus $P$ is extended from $R_{i}$ and so from $R$.

(4.3) CoRollary. Let $R$ be a regular (noetherian) ring such that for every prime integer $p$ either $p$ is a unit in $R$, or $R / p R$ is regular. Then the $B Q$ Conjecture holds for $R$.

(4.4) CoRollary. Let $(R, m)$ be a regular local ring such that either

i) $R$ contains a field, or

ii) $p:=\operatorname{char}(R / \mathfrak{m}) \notin \mathfrak{m}^{2}$.

Then the $B Q$ Conjecture holds for $R$.

\section{REFERENCES}

[1] M. Artin and J. Denef, Smoothing of a ring homomorphism along a section, in: Arithmetic and Geometry II, Birkhäuser, Boston, 1983, 5-32.

[2 ] H. Bass, Some problems in "classical" algebraic $K$-theory, in: Algebraic $K$-theory II, Lecture Notes in Math., Vol. 342, Berlin, 1973, 1-70.

[3] S. M. Bhatwadekar and R. A. Rao, On a question of Quillen, Trans. Amer. Math. Soc., 279 (1983), 801-810.

[4] A. Grothendieck and J. Dieudonné, Eléments de géometrie algébrique IV, Part 1, Publ. Math. IHES, Paris, 1964.

[5] H. Lindel, On the Bass-Quillen Conjecture concerning projective modules over polynomial rings, Invent. Math., 65 (1981), 319-323.

[6] H. Matsumura, Commutative algebra, Benjamin, New York, 1980.

[ 7 ] M. P. Murthy, A letter containing Swan's notes on Lindel's results.

[8] D. Popescu, General Néron desingularization and approximation, Nagoya Math. J., 104 (1986), 85-115.

[9] D. Popescu, On a question of Quillen, to appear.

[10] D. Quillen, Projective modules over polynomial rings, Invent. Math., 36 (1976), 167-171.

[11] M. Raynaud, Anneaux locaux henséliens, Lecture Notes in Math., Vol. 169, Berlin, 1970.

[12] M. Roitman, On projective modules over polynomial rings, J. Algebra, 58 (1979), 51-63.

[13] A. A. Suslin, Projective modules over a polynomial ring are free, Soviet. Math. Dokl., 17 (1976), 1160-1164.

Mathematics Department

INCREST

Bucharest 79622,

Romania 